\title{
STEM EBIC Mapping of the Metal-Insulator Transition in Thin-film $\mathrm{NbO}_{2}$
}

\author{
William A. Hubbard ${ }^{1}$, Toyanath Joshi ${ }^{2}$, Matthew Mecklenburg ${ }^{3}$, Brian Zutter ${ }^{1}$, Pavel Borisov ${ }^{2}$, David \\ Lederman $^{2,4}$, and B. C. Regan ${ }^{1}$ \\ 1. Department of Physics \& Astronomy and California NanoSystems Institute, University of California, \\ Los Angeles, CA, USA \\ 2. Department of Physics and Astronomy, West Virginia University, Morgantown, WV, USA \\ 3. Center for Electron Microscopy and Microanalysis, University of Southern California, Los Angeles, \\ CA, USA \\ 4. Department of Physics, University of California, Santa Cruz, CA, USA
}

A metal-to-insulator transition (MIT) occurs in $\mathrm{NbO}_{2}$ near $1100 \mathrm{~K}$, coincident with a subtle change in its crystal structure $[1,2]$. Because this transition, or a possibly related one, can be induced by applying a local bias, $\mathrm{NbO}_{2}$ could potentially serve as a selector material that reduces the "sneak current" problem in resistive memory arrays. In a previous study [3] we attempted to image the bias-induced transition in $\mathrm{Pt} / \mathrm{NbO}_{2} / \mathrm{Pt}$ devices with scanning transmission electron microscopy (STEM) while switching in situ. The transition-driven structural changes proved to be too subtle to map the transitioned area effectively. Here we report STEM electron beam-induced current (STEM EBIC) imaging of a thermally-induced MIT in thin-film $\mathrm{NbO}_{2}$ devices.

We fabricated a Ti/Pt $(5 / 25 \mathrm{~nm})$ heater on an electron-transparent $\mathrm{Si}_{3} \mathrm{~N}_{4}$ membrane and coated the device with a $20 \mathrm{~nm}$ conformal layer of $\mathrm{Al}_{2} \mathrm{O}_{3}$ using atomic layer deposition (ALD). We then patterned Ti/Pt probe electrodes on the $\mathrm{Al}_{2} \mathrm{O}_{3}$ and coated these with $30 \mathrm{~nm}$ of $\mathrm{NbO}_{2}$ via pulsed laser deposition using a previously reported recipe $[2,3]$. We suspect that, because of air exposure, a thin layer of insulating $\mathrm{Nb}_{2} \mathrm{O}_{5}$ coats the $\mathrm{NbO}_{2}$. Figure 1 shows four STEM images (top row) and a schematic (lower left) of the resultant heating/biasing $\mathrm{NbO}_{2}$ device. A current amplifier is attached to the probes, which are electrically connected to the $\mathrm{NbO}_{2}$. Thus, to the extent that the $\mathrm{NbO}_{2}$ conducts, the current amplifier is connected to the entire $\mathrm{NbO}_{2}$ layer. In contrast, the current amplifier is nominally isolated from the heater by the insulating $\mathrm{Al}_{2} \mathrm{O}_{3}$.

With this heating/biasing device architecture, we measured the resistance between the probes (i.e. through the $\mathrm{NbO}_{2}$ ) and acquired STEM images for a series of heater powers. As the heater power was increased, the resistance between the probes (plotted in green in the lower-right of Figure 1) dropped sharply at first, and then leveled off after $\sim 500 \mu \mathrm{W}$. As the heater power was changed, the first three, standard STEM images showed only minor changes, which we attribute to small, thermally-induced grain rotations. In contrast, the STEM EBIC image developed a large, obvious dark patch (representing a negative EBIC) centered on the region expected to see the largest heating.

The plot in the lower center of Figure 1 shows the raw, average current measured by the current amplifier when the STEM electron beam is in the regions in blue (near the heater) and red (far from the heater) indicated on the EBIC image. These curves are dominated by a beam-position-independent current that is present even when the beam is blanked. This current contribution we attribute to leakage between the $\mathrm{Pt}$ heater and $\mathrm{NbO}_{2}$ film and is, by definition, not an EBIC. The true EBIC signal is taken to be the difference between the two raw signals, and is shown in black in the lower-right plot. This signal is always negative, 
indicating that the $\mathrm{NbO}_{2}$ layer is collecting electrons, regardless of whether the heater bias is positive or negative. The origin of these electrons is not yet well understood.

\section{References:}

[1] AA Bolzan et al, Journal of Solid State Chemistry 113 (1994), p. 9-14.

[2] T Joshi et al, Journal of Physics D: Applied Physics 48 (2015), p. 335308.

[3] WA Hubbard, et al, Microscopy and Microanalysis 22, S3 (2016), p. 1254-55.

[4] This work has been supported by FAME, one of six centers of STARnet, a Semiconductor Research Corporation program sponsored by MARCO and DARPA, by National Science Foundation (NSF) award DMR-1611036, and by NSF STC award DMR-1548924. The authors acknowledge the use of instruments at the Electron Imaging Center for NanoMachines supported by NIH 1S10RR23057 and the CNSI at UCLA, as well as the Shared Research Facilities at WVU.

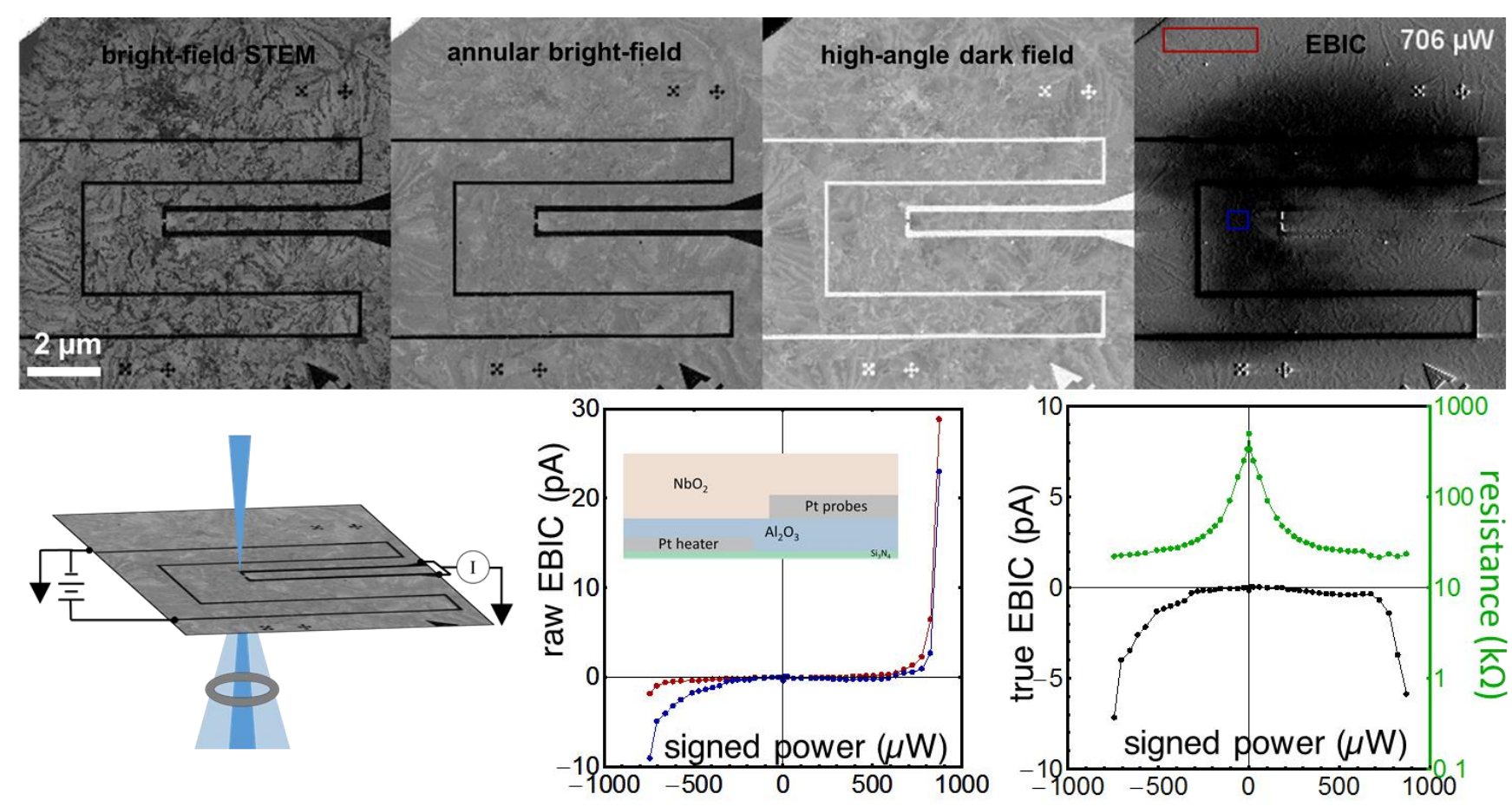

Figure 1. (top row) Three standard STEM images, and one STEM EBIC image, simultaneously acquired with $706 \mu \mathrm{W}$ applied to the heater. A perspective schematic of the experiment (lower left) shows the EBIC amplifier attached to the probes on the right and a voltage source driving the heater on the left. The inset in the lower-center plot shows a cross-sectional schematic of the device. This plot shows average current measured for a region inside (blue) and outside (red) the heater, as indicated on the EBIC image. The difference between the red and blue plots, or the true EBIC signal, is shown in black in the lower-right plot, along with the resistance measured between the probes in green. 